\title{
Comparison of Different Fault Detection Statistics Detectability in PCA
}

\author{
Tian Qiu \\ Key Laboratory of Measurement and Control for Industry \\ Process \\ North China Electric Power University \\ Beijing, China \\ Email: qiutian@ncepu.edu.cn
}

\author{
Xiaojing Bai \\ Key Laboratory of Measurement and Control for Industry \\ Process \\ North China Electric Power University \\ Beijing, China \\ Email: baixiaojing1987@163.com
}

\begin{abstract}
Detectability of the sensor fault detection system is the basic criteria for selecting of different fault detection statistics. $P V R$ and $C V R$ in MPCA are compared with $T_{H}^{2}$. The complementary among them is analyzed qualitatively. Results of power plant data simulation show that $P V R$ and $C V R$ in MPCA can improve sensor fault detection, but $T_{H}^{2}$ is still better for some sensors. Combination of $P V R, C V R$ and $T_{H}^{2}$ can improve sensor fault detectability of PCA-based fault detection system.
\end{abstract}

Keywords-process monitoring, fault detection, Principal component analysis

\section{INTRODUCTION}

Process monitoring in power plants need operators to monitor a considerable amount of process variables. Current methods usually set the upper and lower limit of variables to realize simple monitor. This kind of methods cannot change the upper and lower limit according to the operation condition automaticly, so the faults with little magnitude cannot be detected, which is detrimental to early fault detection and cannot satisfy the increasingly strict monitoring requirement. Multivariable analysis methods build process models with the correlation among process variables. These kind of methods has better performance in fault detection, when the correlation among process variables has changed [1]. In multivariable analysis methods, Principal Component Analysis based sensor fault detection has been researched deeply and applied into different industrial process monitoring [2-12].

$S P E$ (Square Prediction Error) and Hotelling $T^{2}$ are important indice in PCA for fault detection. Dunia et al [2] proposed a whole set PCA method for sensor fault detection, diagnosis and reconstruction using $S P E$. Wang et al [3] analyzed the performance of $T^{2}$ for fault detection based on PCA, and proposed the method of fault diagnosis and reconstruction based on $T^{2}$. Qin [4] summarized fault detecion based on PCA. SPE statitic has different detectability for different sensors [5], Wang et al [6] divided $S P E$ into two statistics PV (Principal-component-related Variable) and CV (Common Variable), and proposed an improvement for classic PCA method to improve the fault detectability.
Hawkins $T_{H}^{2}$ was considered to be similar with SPE. They have different fault detectability for different sensors in fault detetion [7]. It is necessary to investiage the fault detectability among Hawkins $T_{H}^{2}, P V R$ and $C V R$ in improved PCA method, for improved PCA is consided to have been improved the fault detectability of PCA method.

\section{PCA THEORY}

Let $x \in R^{m}$ denote a vector of $\mathrm{m}$ sensors. Assume $X \in R^{N \times m}$ is composed by $\mathrm{N}$ samples of each sensor, and each row represent a sample. According to PCA theory, $\mathrm{x}$ can be divided into 2 parts:

$x=\hat{x}+\tilde{x}$

$\hat{x}$ and $\tilde{x}$ represent the modeling part and residual part separately.

$x$ can be projected to the PCS(Priciple Component Subspace) and then projected back, the result part is defined as $\hat{x}$ :

$\hat{x}=P P^{T} x$

$P \in R^{m \times l}$ is the loading matrix in PCA, which is composed by the if first eigenvector of the samples's covariant matrix. Sample vectors has more variation at these directions, which shows that sample variants have more correlation at these directions. $l<1$ is the dimension of the PCS, the chosen of $l$ depend on the correlation among sample variants. If $l$ is assigned to $m, P P^{T}=I$, sample vector has no information loss in PCA.

$x f$ can be projected to the RS(Residual Subspace) and then projected back, the result part is defined as $f \tilde{x}$ :

$x=\left(I-P P^{T}\right) x$

$\left(I-P P^{T}\right)$ is the residual matrix in PCA, which is composed by the Residual eigenvector of the samples's covariant matrix. Sample vectors have less variance at these directions, which shows that sapmle variants have less correlation at these directions. And the variance at these directions is often consided as noise.

Let consider the situation in which only one sensor has malfunction. When the sensor has fault, the correlation among sample variants is changing. Original PCA model doesn't fit, as $28 \tilde{x}$ is increasing, which means the projection of sample vector to RS is increasing. $S P E$ can be used to detect this kind of fault:

$S P E=\|\tilde{x}\|^{2}<\delta_{S P E}$

The calculation of $\delta_{S P E}$ is described in paper [13]. When $S P E$ is bigger than the control limit, some sensor is 
considered to have fault, on the other hand, every sensor in good condition.

Wang et al [6] proposed an improved PCA method, in which two indice are proposed using multiple correlation among sensors and the principal components. PVR (PV Residuals) is defined by the first $s$ process variables (Principal-component-related Variable, PV), which have remarkable correlation with principalm components; $C 28 V R$ is defined by the rest process variables (Common Variable, $\mathrm{CV})$. These two indice can be calculated as:

$$
\begin{aligned}
& P V R=x_{s}\left(I-P_{s} P_{s}^{T}\right) x_{s}^{T} \\
& C V R=x_{m-s}\left(I-P_{m-s} P_{m-s}^{T}\right) x_{m-s}^{T}
\end{aligned}
$$

Subscript $s 2$ and $m-s$ in $x f$ and $P$ represent PV and $\mathrm{CV}$ part in data vector $x$ and loading matrix $P$ seperately. The control limit of $P V R$ and $C V R$ can be calculated with the description in paper [6].

Hawkins' $T_{H}^{2}$ is defined as [14]:

$$
T_{H}^{2}=\sum_{i=l+1}^{m} \frac{t_{i}^{2}}{\lambda_{i}}
$$

$\tilde{\Lambda}=\operatorname{diag}\left(\lambda_{l+1}, \ldots, \lambda_{m}\right)$ is the diagonal matrix composed with the last $m-l$ eigenvalue of the samples's covariance matix. The control limit can be calculated accroding to the description in paper [14].

\section{COMPARISON BETWEEN $T_{H}^{2}$ AND $P V R / C V R$}

Feng et al [5] pointed out that $S P E$ has different fault detectability with different sensors. $T_{H}^{2}$ and $S P E$ are indices measuring the projections of process variables in the residual subspace, which means $T_{H}^{2}$ and $S P E$ can be compared directly in the residual subspace. The essence of improved PCA method is to decompose $S P E$ into two indice [6], so PV and CV indice should have different fault detectability too. For PV and $\mathrm{CV}$ indice have different variable subspace, compared with $T_{H}^{2}$, they cannot be compared directly, so the analysis has to be qualitative in the following part to compare $P V R$ and $C V R$ with $T_{H}^{2}$ and $S P E$.

$\mathrm{PV}$ and $\mathrm{CV}$ are the $S P E$ in partial variable space, these two indice should have similar properties as $S P E$, so the difference between $S P E$ and $T_{H}^{2}$ is discussed here mainly. $S P E$ and $T_{H}^{2}$ have different fault detectability with different sensors, because their control limits $\delta_{S P E}$ and $\delta_{H}$ have some kind of conservation [7].

The simulation example in paper [4] is applied here for qualitative analysis:

$$
\left[\begin{array}{l}
x_{1} \\
x_{2} \\
x_{3} \\
x_{4}
\end{array}\right]=\left[\begin{array}{rr}
0.3873 & 0.1190 \\
-0.1291 & 0.2379 \\
0.9037 & -0.1530 \\
0.1291 & 0.9518
\end{array}\right]\left[\begin{array}{l}
s_{1} \\
s_{2}
\end{array}\right]+\text { noise }
$$

$x_{i}$ is the measured variable, $s_{1}$ and $s_{2}$ are zero-mean random sequences with standard deviations of 1 , noise is the white noise, whose standard deviations are $0.1,0.8,0.1,0.4$ for $x_{1}, \ldots, x_{4}$ seperately.

1000 samples are generated with the above equation. The PCA model is built with the samples, the dimension of principal subspace is 2. According to MPCA method, $x_{1}$ and $x_{3}$ are the $\mathrm{PV}$, the rest variables are $\mathrm{CV}$. Calculate the indices with $99 \%$ confidence coefficient, the result in shown in Figure 1.

Blue points in Figure 1 represent the projection of data vector to the residual subspace. In the residual subspace, with the coordinates of $t_{3}$ and $t_{4}$, the control limit of SPE is a circle, while the control limit of $T_{H}^{2}$ is a ellipse. Figure 1 (a) shows that the control limit of $S P E$ and $T_{H}^{2}$ can envelope sample data, and $T_{H}^{2}$ 's ellipse shape of the control limit is better than $S P E^{\prime}$ 's to fit the sample data. Figure 1 (b) shows the projection direction of different sensor's fault, the directions of $x_{1}$ and $x_{3}$ are closer to $t_{4}$ coordinate, the directions of $x_{2}$ and $x_{4}$ are closer to $t_{3}$ coordinate. And only fault in $x_{2}$ can cross the control limit of $S P E$ first, $T_{H}^{2}$ can detect other sensors' fault better than $S P E$. This result is consistent with the analysis of Feng et al in paper [5].

Figure 1 (c) and (d) shows that the control limit of $P V R$ evelopes the sample data set well, while the control limit of $C V R$ leaves a few sample datas outside of the limit, which indicates that MPCA maybe too strict to keep false alarm rate as small as declared by the confidence coefficient.

The conservation SPE statstic represents process data have different divergent in different direction in RS, while the control limit of $\delta_{S P E}$ is the same value at different direction. Improved PCA method decomposes $S P E$ into 2 indice, which decrease the conservation of $S P E$ in some degree, but $P V R$ and $C V R$ still have similar conservation as $S P E$. Improved PCA method should be able to improve the fault Detectability, but $T_{H}^{2}$ cannot be replaced.

\section{POWER PLANT DATA SIMULATION}

Thermal process data is retrieved from a $215 \mathrm{MW}$ power plant to build PCA model, and sensor fault is simulated for fault detection testing.

According to the correlation analysis of the data, 10 variables are selected, such as feedwater pressure, lowpressure cylinder exhausting pressure, main steam flow rate, pressure behind feedwater valve, pressure before feedwater valve, reheater steam temperature, circulating water temperature I, circulating water temperature II, superheater spray flow rate, reheater spray flow rate.

Two sets of process data are retrieved when thermal process is in stable condition. The first data set which has 700 samples is used as sample data for modeling. The second data set which has 60 samples was added with fault for verifying the fault detectability of different indices. The fault was added from the 10th sample in the second data set.

The first data set was used to build PCA model, 3 principal components was seleted for their contribution rate is $84 \%$.

According to the improved PCA method, PV variables are selected for the multiple correlation coefficient is bigger than 0.85 and the absolute value of the correlation coefficient is bigger than 0.1. $\left\{x_{1}, x_{2}, x_{6}, x_{7}, x_{8}\right\}$ is selected as PV variables, the rest sensors are considered as $\mathrm{CV}$ variables. The threshold value of different fault detection indices is listed in Table.1 (NOTE: $P V R$ and $C V R$ can only 
be calculated to different sensors, so $\left|f_{k}\right|_{P V R \mathrm{~V}}$ represents the thresholds of fault detection for both $P V R$ and $C V R$ ).

Compared with $S P E$, the fault detectability of improved PCA method has been improved as shown in Table.1, except $x_{6}$. To the most sensors, $\left\{x_{1}, x_{2}, x_{4}, x_{5}, x_{7}, x_{8}\right\}, T_{H}^{2}$ 's fault detectable threshold is smaller than $S P E, P V R$ and $C V R$, which means that $T_{H}^{2}$ has better fault detectability than the other indices. Bias faults, 0.4 and 1.1, are introduced into $x_{7}$, as condition I and II. Fault detection results are shown in Figure 2. According to improved PCA method, $x_{7}$ belongs to high multiple correlation coefficient sensors, $P V R$ should be used for fault detecion.

Figure 2 shows: $S P E$ can only detect a few fault to bigger bias fault; $P V R, C V R$ and $T_{H}^{2}$ detect the bigger bias fault just at the sample where bias fault has been introduced; $S P E, P V R$ and $C V R$ indices can not detect the smaller bias fault; $T_{H}^{2}$ can not only detect bigger bias fault, but also give sustaining alarm for smaller bias fault. This proves that $S P E, P V R, C V R$ and $T_{H}^{2}$ do have different detectaility for different sensors, which means these indices should be combined to detect fault in process monitoring.

\section{SUMMARIES}

The fault detectabilities of $S P E, P V R / C V R, T_{H}^{2}$ indices are analyzed qualitatively and quantitatively. According to the qualitative analysis, $S P E, P V R / C V R$ and $T_{H}^{2}$ indices have different fault detectability to different sensors. According to the quantitative analysis, $P V R / C V R$ have better fault detectibility than $S P E$, while $T_{H}^{2}$ has better result in partial sensors than $P V R / C V R$, which means $T_{H}^{2}$ should be combined in the fault detection system based on PCA. The qualitative analysis of $P V R$ and $C V R$ shows that the control limit of $C V R$ is too small to envelope most sample datas in it. This indicates MPCA-based fault detection system has large false alarm rate, which need further reaserach to confirm.

\section{ACKNOLEDGEMENT}

This work was supported by "The Fundamental Research Funds for the Central Universities"

\section{REFERENCES}

[1] Kourti T.; MacGregor J. F.. Process Analysis, Monitoring and Diagnosis, Using Multivariate Projection Methods. Chemometrics and Intelligent Laboratory Systems [J], 1995, 28 (1), PP:3-21.

[2] Dunia R.; Qin S. J.; Edgar T. F. et al. Identification of Faulty Sensors Using Principal Component Analysis. AIChE Journal [J], 1996, 42 (10), PP:2797-2812.

[3] Wang H.; Song Z.; Li P.. Fault Detection Behavior and Performance Analysis of Principal Component Analysis Based Process Monitoring Methods. Ind Eng Chem Res [J], 2002, 41 (10), PP:2455-2464.

[4] Qin S. J.. Statistical Process Monitoring: Basics and Beyond. Journal of Chemometrics [J], 2003, 17(8-9), PP:480-520.

[5] Feng X.; Yang, X.; Xu, Y.. Squared Prediction Error Analysis of Multivariate Statistical Process Control. Journal of Tsinghua University (Science and Technology) [J], 1999, 39(7), PP:41-45.

[6] Wang H.; Song Z.; Li P.. Improved PCA with Application to Process Monitoring and Fault Diagnosis. Journal of Chemical Industry and Engineering (China) [J], 2001, 52(6), PP:471-475.

[7] Qiu T.; Ding Y.; Wu Z.. Sensor Fault Detection Statistics Based on Principal Component Analysis. Journal of Tsinghua University (Science and Technology) [J], 2006, 46(8), PP:1147-1450.

[8] Huang X.; Niu Z.; Niu Y. et al. Behavior Analysis of Sensor Fault Detection Using PCA Approach. Ind Eng Chem Res [J], 2003, 16 (4), PP:419-423.

[9] Huang X.; Liu J.; Niu Y.. Application of PCA-based Fault Detection in Thermal Boiler Processes. Power Engineering [J], 2004, 24(4), PP:542-547.

[10] Dunia R.; Qin S. J.; Edgar T. F. et al. Use of Principal Component Analysis for Sensor Fault Identification. Computers and Chemical Engineering [J], 1996, 20(Suppl.), PP:S713-S718.

[11] Wang H.; Song Z.; Li P.. Study on the Fault Detectability of Principal Component Analysis. Chinese Journal of Scientific Instrument [J], 2002, 23(3), PP:232-240.

[12] Wang H.; Song Z.; Wang H.. Fault Detection Behavior Analysis of PCA-based Process Monitoring Approach. Journal of Chemical Industry and Engineering (China) [J], 2002, 53 (3), PP:297-301.

[13] Jackson J. E.; Mudholkar G. S.. Control Procedures for Residuals Associated with Principal Component Analysis. Technometrics [J], 1979, 21(3), PP:341-349.

[14] Jackson J. E.. A User's Guide to Principal Components [M], 2003, John Wiley \& Sons Inc.
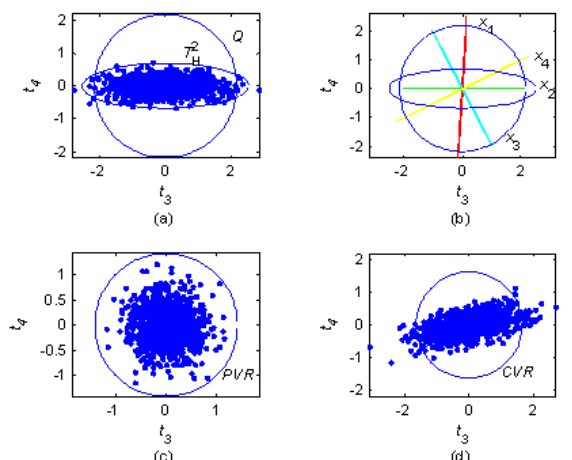

Figure 1 Comparison among the control limits of $S P E, P V R, C V R$ and $T_{H}^{2}$ 

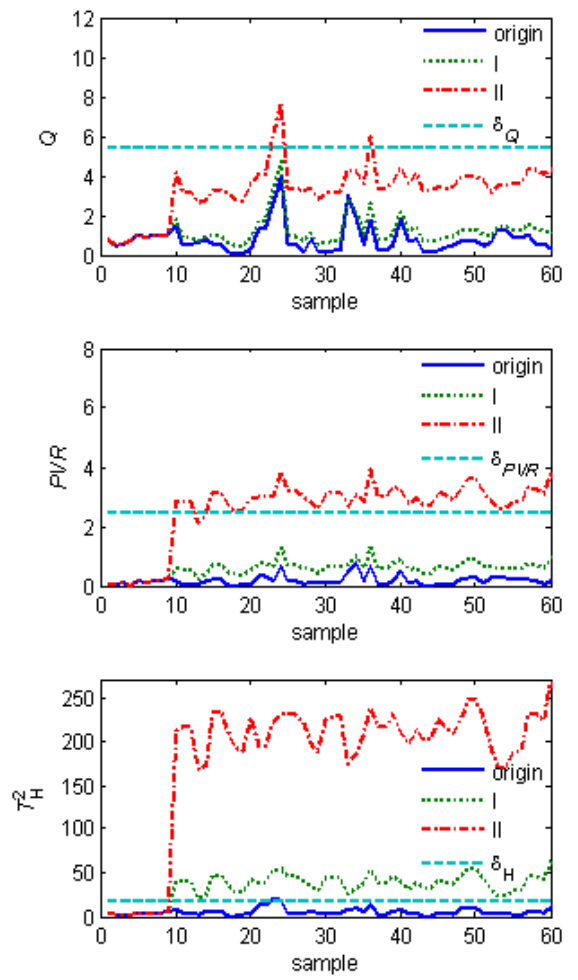

Figure 2 Fault detection result for bias fault

TABLE I. THRESHOLDS UNDER NECESSARY CONDITION FOR FAULT DETECTION

\begin{tabular}{crrr}
\hline sensors & \multicolumn{1}{c}{$\left|f_{k}\right|$ SPE } & \multicolumn{1}{c}{$\left|f_{k}\right|_{P V R}$} & \multicolumn{1}{c}{$\left|f_{k}\right|_{H}$} \\
\hline $\mathrm{X}_{1}$ & 2.2526 & 1.1947 & 0.9386 \\
$\mathrm{X}_{2}$ & 4.8559 & 3.2442 & 0.4499 \\
$\mathrm{X}_{3}$ & 66.3125 & 41.1624 & 41.2026 \\
$\mathrm{X}_{4}$ & 0.5476 & 0.3352 & 0.3307 \\
$\mathrm{x}_{5}$ & 1.5447 & 0.8181 & 0.5609 \\
$\mathrm{x}_{6}$ & 35.2096 & 77.1744 & 39.5725 \\
$\mathrm{x}_{7}$ & 8.6739 & 4.6090 & 0.4296 \\
$\mathrm{X}_{8}$ & 8.2347 & 4.3656 & 0.6236 \\
$\mathrm{X}_{9}$ & 33.0671 & 21.3747 & 38.563 \\
$\mathrm{X}_{10}$ & 2.3499 & 1.6309 & 3.3966 \\
\hline
\end{tabular}

